\title{
APPENDIX I. CONCORDANCE OF UNDERTEXT AND OVERTEXT PAGES
}

\begin{tabular}{|l|l|}
\hline Undertext & Overtext \\
\hline Ir (top) & $90 \mathrm{v}$ \\
\hline Ir (bottom) & $95 \mathrm{r}$ \\
\hline Iv (top) & $90 \mathrm{r}$ (pencil 89) \\
\hline Iv (bottom) & $95 \mathrm{r}$ \\
\hline IIr (top) & $44 \mathrm{r}$ (pencil 43) \\
\hline IIr (bottom) & $45 \mathrm{v}$ \\
\hline IIv (top) & $44 \mathrm{v}$ \\
\hline IIv (bottom) & $45 \mathrm{r}$ (pencil 44) \\
\hline IIIr (top) & $107 \mathrm{r}$ (pencil 106) \\
\hline IIIr (bottom) & $110 \mathrm{v}$ \\
\hline IIIv (top) & $107 \mathrm{v}$ \\
\hline IIIv (bottom) & $110 \mathrm{r}$ (pencil 109) \\
\hline IVr (top) & $170 \mathrm{r}$ (pencil 169) \\
\hline IVr (bottom) & $175 \mathrm{v}$ \\
\hline IVv (top) & $170 \mathrm{v}$ \\
\hline IVv (bottom) & $175 \mathrm{r}$ (pencil 174) \\
\hline Vr (top) & $81 \mathrm{r}$ (pencil 80) \\
\hline Vr (bottom) & $88 \mathrm{v}$ \\
\hline Vv (top) & $81 \mathrm{v}$ \\
\hline Vv (bottom) & $88 \mathrm{r}$ (pencil 87) \\
\hline VIr (top) & $52 \mathrm{r}$ (pencil 51) \\
\hline VIr (bottom) & $53 \mathrm{v}$ \\
\hline VIv (top) & $52 \mathrm{v}$ \\
\hline VIv (bottom) & $53 \mathrm{r}$ (pencil 52) \\
\hline
\end{tabular}

\begin{tabular}{|l|l|}
\hline Undertext & Overtext \\
\hline $\begin{array}{l}\text { VIIr (bottom; top } \\
\text { missing, sewn onto } \\
\text { LXXXIXr) }\end{array}$ & $169 \mathrm{r}$ (pencil 168) \\
\hline $\begin{array}{l}\text { VIIv (bottom; top } \\
\text { missing, sewn onto } \\
\text { LXXIXv) }\end{array}$ & $169 \mathrm{v}$ \\
\hline VIIIr (top) & $108 \mathrm{r}$ (pencil 107) \\
\hline VIIIr (bottom) & $109 \mathrm{v}$ \\
\hline VIIIv (top) & $108 \mathrm{v}$ \\
\hline VIIIv (bottom) & $109 \mathrm{r}$ (pencil 108) \\
\hline IXr (top) & $132 \mathrm{r}$ (pencil 131) \\
\hline IXr (bottom) & $133 \mathrm{v}$ \\
\hline IXv (top) & $132 \mathrm{v}$ \\
\hline IXv (bottom) & $133 \mathrm{r}$ (pencil 132) \\
\hline Xr (top) & $77 \mathrm{r}$ (pencil 76) \\
\hline Xr (bottom) & $76 \mathrm{v}$ \\
\hline Xv (top) & $77 \mathrm{v}$ \\
\hline Xv (bottom) & $76 \mathrm{r}$ (pencil 75) \\
\hline XIr (top) & $16 \mathrm{r}$ \\
\hline XIr (bottom) & $9 \mathrm{v}$ \\
\hline XIv (top) & $16 \mathrm{v}$ \\
\hline XIv (bottom) & $33 \mathrm{r}$ (pencil 32) \\
\hline XIIr (top) & $33 \mathrm{v}$ \\
\hline XIIr (bottom) & \\
\hline XIIv (top) & \\
\hline
\end{tabular}




\begin{tabular}{|l|l|}
\hline Undertext & Overtext \\
\hline XIIv (bottom) & $40 \mathrm{r}$ (pencil 39) \\
\hline XIIIr (top) & $18 \mathrm{r}$ \\
\hline XIIIr (bottom) & $23 \mathrm{v}$ \\
\hline XIIIv (top) & $18 \mathrm{v}$ \\
\hline XIIIv (bottom) & $\begin{array}{l}23 \mathrm{r} \text { (pencil 22 } \\
\text { corrected to 23) }\end{array}$ \\
\hline XIVr (top) & $131 \mathrm{r}$ (pencil 130) \\
\hline XIVr (bottom) & $134 \mathrm{v}$ \\
\hline XIVv (top) & $131 \mathrm{v}$ \\
\hline XIVv (bottom) & $134 \mathrm{r}$ (pencil 133) \\
\hline XVr (top) & $51 \mathrm{r}$ (pencil 50) \\
\hline XVr (bottom) & $54 \mathrm{v}$ \\
\hline XVv (top) & $51 \mathrm{v}$ \\
\hline XVv (bottom) & $54 \mathrm{r}$ (pencil 53) \\
\hline XVIr (top) & $20 \mathrm{r}$ \\
\hline XVIr (bottom) & $21 \mathrm{v}$ \\
\hline XVIV (top) & $20 \mathrm{v}$ \\
\hline XVIv (bottom) & $21 \mathrm{r}$ \\
\hline XVIIr (top) & $117 \mathrm{r}$ (pencil 116) \\
\hline XVIIr (bottom) & $116 \mathrm{v}$ \\
\hline XVIIv (top) & $117 \mathrm{v}$ \\
\hline XVIIv (bottom) & $116 \mathrm{r}$ (pencil 115) \\
\hline XVIIIr (top) & $140 \mathrm{r}$ (pencil 139) \\
\hline XVIIIr (bottom) & $141 \mathrm{v}$ \\
\hline XVIIIv (top) & $140 \mathrm{v}$ \\
\hline XVIIIv (bottom) & $141 \mathrm{r}$ (pencil 140) \\
\hline XIXr (top) & $118 \mathrm{r}$ (pencil 117) \\
\hline XIXr (bottom) & $115 \mathrm{v}$ \\
\hline XIXv (top) & $118 \mathrm{v}$ \\
\hline XIXv (bottom) (pencil 40) \\
\hline XXr (top) & \\
\hline XXr (bottom) & 114 ) \\
\hline
\end{tabular}

\begin{tabular}{|l|l|}
\hline Undertext & Overtext \\
\hline XXV (top) & $41 \mathrm{v}$ \\
\hline XXv (bottom) & $48 \mathrm{r}$ (pencil 47) \\
\hline XXIr (top) & $101 \mathrm{r}$ (pencil 100) \\
\hline XXIr (bottom) & $100 \mathrm{v}$ \\
\hline XXIv (top) & $101 \mathrm{v}$ \\
\hline XXIv (bottom) & $100 \mathrm{r}$ (pencil 99) \\
\hline XXIIr (top) & $126 \mathrm{r}$ (pencil 125) \\
\hline XXIIr (bottom) & $123 \mathrm{v}$ \\
\hline XXIIv (top) & $126 \mathrm{v}$ \\
\hline XXIIv (bottom) & $123 \mathrm{r}$ (pencil 122) \\
\hline XXIIIr (top) & $125 \mathrm{r}$ (pencil 124) \\
\hline XXIIIr (bottom) & $124 \mathrm{v}$ \\
\hline XXIIIv (top) & $125 \mathrm{v}$ \\
\hline XXIIIv (bottom) & $124 \mathrm{r}$ (pencil 123) \\
\hline XXIVr (top) & $163 \mathrm{r}$ (pencil \\
\hline XXIVr (bottom) & 162 ) \\
\hline XXIVv (top) & $163 \mathrm{v}$ \\
\hline XXIVv (bottom) & $166 \mathrm{r}$ (pencil 165) \\
\hline XXVr (top) & $10 \mathrm{r}$ \\
\hline XXVr (bottom) & $15 \mathrm{v}$ \\
\hline XXVv (top) & $10 \mathrm{v}$ \\
\hline XXVv (bottom) & $15 \mathrm{r}$ \\
\hline XXVIr (top) & $37 \mathrm{r}$ (pencil 36) \\
\hline XXVIr (bottom) & $36 \mathrm{v}$ \\
\hline XXVIv (top) & $37 \mathrm{v}$ \\
\hline XXVIv (bottom) & $36 \mathrm{r}$ (pencil 35) \\
\hline XXVIIr (top) & $56 \mathrm{r}$ (pencil 55) \\
\hline XXVIIr (bottom) & $49 \mathrm{v}$ \\
\hline XXVIIv (top) & $56 \mathrm{v}$ \\
\hline XXVIIv (bottom) & $49 \mathrm{r}$ (pencil 48) \\
\hline XXVIIIr (top) & $119 \mathrm{r}$ (pencil 118) \\
\hline
\end{tabular}




\begin{tabular}{|l|l|}
\hline Undertext & Overtext \\
\hline XXVIIIr (bottom) & $114 \mathrm{v}$ \\
\hline XXVIII (top) & $119 \mathrm{v}$ \\
\hline XXVIIIv (bottom) & $114 \mathrm{r}$ (pencil 113) \\
\hline XXIXr (top) & $120 \mathrm{r}$ (pencil 119) \\
\hline XXIXr (bottom) & $113 \mathrm{v}$ \\
\hline XXIXv (top) & $120 \mathrm{v}$ \\
\hline XXIXv (bottom) & $113 \mathrm{r}$ (pencil 112) \\
\hline XXXr (top) & $55 \mathrm{r}$ (pencil 54) \\
\hline XXXr (bottom) & $50 \mathrm{v}$ \\
\hline XXXv (top) & $55 \mathrm{v}$ \\
\hline XXXV (bottom) & $50 \mathrm{r}$ (pencil 49) \\
\hline XXXIr (top) & $61 \mathrm{r}$ (pencil 60) \\
\hline XXXIr (bottom) & $60 \mathrm{v}$ \\
\hline XXXIv (top) & $61 \mathrm{v}$ \\
\hline XXXIV (bottom) & $60 \mathrm{r}$ (pencil 59) \\
\hline XXXIIr (top) & $146 \mathrm{r}$ (pencil 145) \\
\hline XXXIIr (bottom) & $151 \mathrm{v}$ \\
\hline XXXIIv (top) & $146 \mathrm{v}$ \\
\hline XXXIIv (bottom) & $151 \mathrm{r}$ (pencil 150) \\
\hline XXXIIIr (top) & $145 \mathrm{r}$ (pencil 144) \\
\hline XXXIIIr (bottom) & $152 \mathrm{v}$ \\
\hline XXXIIIv (top) & $145 \mathrm{v}$ \\
\hline XXXIIIv (bottom) & $152 \mathrm{r}$ (pencil 151) \\
\hline XXXIVr (top) & $78 \mathrm{r}$ (pencil 77) \\
\hline XXXIVr (bottom) & $75 \mathrm{v}$ \\
\hline XXXIVv (top) & $78 \mathrm{v}$ \\
\hline XXXIVv (bottom) & $75 \mathrm{r}$ (pencil 74) \\
\hline XXXVr (top) & $156 \mathrm{r}$ (pencil 155) \\
\hline XXXVr (bottom) & $157 \mathrm{v}$ \\
\hline XXXVv (top) & $156 \mathrm{v}$ \\
\hline XXXVv (bottom) & $157 \mathrm{r}$ pencil 156) \\
\hline XXXVIr (top) & 66 ) \\
\hline
\end{tabular}

\begin{tabular}{|c|c|}
\hline Undertext & Overtext \\
\hline XXXVIr (bottom) & 70r (pencil 69) \\
\hline XXXVIV (top) & $67 \mathrm{v}$ \\
\hline XXXVIv (bottom) & $70 \mathrm{v}$ \\
\hline XXXVIIr (top) & 46r (pencil 45) \\
\hline XXXVIIr (bottom) & $43 v$ \\
\hline XXXVIIV (top) & $46 \mathrm{v}$ \\
\hline XXXVIIv (bottom) & 43r (pencil 42) \\
\hline XXXVIIIr (top) & 97r (pencil 96) \\
\hline XXXVIIIr (bottom) & $104 \mathrm{v}$ \\
\hline XXXVIIIv (top) & $97 \mathrm{v}$ \\
\hline XXXVIIIV (bottom) & 104r (pencil 103) \\
\hline XXXIXr (top) & 122r(pencil 121) \\
\hline XXXIXr (bottom) & $127 \mathrm{v}$ \\
\hline XXXIXv (top) & $122 \mathrm{v}$ \\
\hline XXXIXv (bottom) & 127r(pencil 126) \\
\hline $\mathrm{XLr}$ (top) & 121r (pencil 120) \\
\hline XLr (bottom) & $128 \mathrm{v}$ \\
\hline XLv (top) & $121 \mathrm{v}$ \\
\hline XLv (bottom) & 128r (pencil 127) \\
\hline XLIr (top) & 98r (pencil 97) \\
\hline XLIr (bottom) & $103 \mathrm{v}$ \\
\hline XLIv (top) & $98 \mathrm{v}$ \\
\hline XLIv (bottom) & 103r (pencil 102) \\
\hline XLIIr (top) & 47r (pencil 46) \\
\hline XLIIr (bottom) & $42 \mathrm{v}$ \\
\hline XLIIv (top) & $47 \mathrm{v}$ \\
\hline XLIIv (bottom) & $42 \mathrm{r}($ pencil 41) \\
\hline XLIIIr (top) & 68r (pencil 67) \\
\hline XLIIIr (bottom) & $69 \mathrm{v}$ \\
\hline XLIIIV (top) & $68 \mathrm{v}$ \\
\hline XLIIIv (bottom) & 69r (pencil 68) \\
\hline XLIVr (top) & $71 \mathrm{v}$ \\
\hline
\end{tabular}




\begin{tabular}{|l|l|}
\hline Undertext & Overtext \\
\hline XLIVr (bottom) & $66 \mathrm{r}$ (pencil 65) \\
\hline XLIVv (top) & $71 \mathrm{r}$ (pencil 70) \\
\hline XLIVv (bottom) & $66 \mathrm{v}$ \\
\hline XLVr (top) & $153 \mathrm{r}$ (pencil 152) \\
\hline XLVr (bottom) & $160 \mathrm{v}$ \\
\hline XLVv (top) & $153 \mathrm{v}$ \\
\hline XLVv (bottom) & $160 \mathrm{r}$ (pencil 159) \\
\hline XLVIr (top) & $74 \mathrm{r}$ (pencil 73) \\
\hline XLVIr (bottom) & $79 \mathrm{v}$ \\
\hline XLVIv (top) & $74 \mathrm{v}$ \\
\hline XLVIv (bottom) & $79 \mathrm{r}$ (pencil 78) \\
\hline XLVIIr (top) & $73 \mathrm{r}$ (pencil 72) \\
\hline XLVIIr (bottom) & $80 \mathrm{v}$ \\
\hline XLVIIv (top) & $73 \mathrm{v}$ \\
\hline XLVIIv (bottom) & $80 \mathrm{r}$ (pencil 79) \\
\hline XLVIIIr (top) & $154 \mathrm{r}$ (pencil 153) \\
\hline XLVIIIr (bottom) & $159 \mathrm{v}$ \\
\hline XLVIIIv (top) & $154 \mathrm{v}$ \\
\hline XLVIIIv (bottom) & $159 \mathrm{r}$ (pencil 158) \\
\hline XLIXr (top) & $65 \mathrm{r}$ (pencil 64) \\
\hline XLIXr (bottom) & $72 \mathrm{v}$ \\
\hline XLIXv (top) & $65 \mathrm{v}$ \\
\hline XLIXv (bottom) & $72 \mathrm{r}$ (71 pencil) \\
\hline Lr (top) & $19 \mathrm{r}$ \\
\hline Lr (bottom) & $22 \mathrm{v}$ \\
\hline Lv (top) & $19 \mathrm{v}$ \\
\hline Lv (bottom) & $92 \mathrm{r}$ (pencil 21 \\
\hline LIr (top) \\
\hline LIr (bottom) \\
\hline LIv (top) & \\
\hline LIv (bottom) & \\
\hline
\end{tabular}

\begin{tabular}{|l|l|}
\hline Undertext & Overtext \\
\hline LIIr (top) & $86 \mathrm{r}$ (pencil 85) \\
\hline LIIr (bottom) & $83 \mathrm{v}$ \\
\hline LIIv (top) & $86 \mathrm{v}$ \\
\hline LIIv (bottom) & $83 \mathrm{r}$ (pencil 82) \\
\hline LIIIr (top) & $87 \mathrm{r}$ (pencil 86) \\
\hline LIIIr (bottom) & $82 \mathrm{v}$ \\
\hline LIIIv (top) & $87 \mathrm{v}$ \\
\hline LIIIv (bottom) & $82 \mathrm{r}$ (pencil 81) \\
\hline LIVr (top) & $138 \mathrm{r}$ (pencil 137) \\
\hline LIVr (bottom) & $143 \mathrm{v}$ \\
\hline LIVv (top) & $138 \mathrm{v}$ \\
\hline LIVv (bottom) & $143 \mathrm{r}$ (pencil 142) \\
\hline LVr (top) & $24 \mathrm{r}$ (pencil 23 \\
\hline corrected to 24) \\
\hline LVr (bottom) & $17 \mathrm{v}$ \\
\hline LVv (top) & $24 \mathrm{v}$ \\
\hline LVv (bottom) & $17 \mathrm{r}$ \\
\hline LVIr (top) & $1 \mathrm{r}$ \\
\hline LVIr (bottom) & $8 \mathrm{v}$ \\
\hline LVIv (top) & $1 \mathrm{v}$ \\
\hline LVIv (bottom) & $8 \mathrm{r}$ \\
\hline LVIIr (top) & $38 \mathrm{r}$ (pencil 37) \\
\hline LVIIr (bottom) & $35 \mathrm{v}$ \\
\hline LVIIv (top) & $38 \mathrm{v}$ \\
\hline LVIIv (bottom) & $35 \mathrm{r}$ (pencil 34) \\
\hline LXIIIr (top) & $3 \mathrm{r}$ \\
\hline LVIIIr (top) & $39 \mathrm{r}$ (pencil 38) \\
\hline LXIIIr (bottom) & $6 \mathrm{v}$ \\
\hline LVIIIr (bottom) & $34 \mathrm{v}$ \\
\hline LXIIIv (top) & $3 \mathrm{v}$ \\
\hline LVIIIv (top) & $39 \mathrm{v}$ \\
\hline LXIIIv (bottom) & $6 \mathrm{r}$ \\
\hline
\end{tabular}




\begin{tabular}{|l|l|}
\hline Undertext & Overtext \\
\hline LVIIIv (bottom) & $34 \mathrm{r}$ (pencil 33) \\
\hline LIXr (top) & $94 \mathrm{v}$ \\
\hline LIXr (bottom) & $91 \mathrm{r}$ (pencil 90) \\
\hline LIXv (top) & $94 \mathrm{r}$ (pencil 93) \\
\hline LIXV (bottom) & $91 \mathrm{v}$ \\
\hline LXr (top) & $4 \mathrm{r}$ \\
\hline LXr (bottom) & $5 \mathrm{v}$ \\
\hline LXV (top) & $4 \mathrm{v}$ \\
\hline LXv (bottom) & $5 \mathrm{r}$ \\
\hline LXIr (top) & $57 \mathrm{r}$ (pencil 56) \\
\hline LXIr (bottom) & $64 \mathrm{v}$ \\
\hline LXIv (top) & $57 \mathrm{v}$ \\
\hline LXIv (bottom) & $64 \mathrm{r}$ (pencil 63) \\
\hline LXIIr (top) & $150 \mathrm{r}$ (pencil 149) \\
\hline LXIIr (bottom) & $147 \mathrm{v}$ \\
\hline LXIIv (top) & $150 \mathrm{v}$ \\
\hline LXIIv (bottom) & $147 \mathrm{r}$ (pencil 146) \\
\hline LXIVr (top) & $144 \mathrm{r}$ (pencil 143) \\
\hline LXIVr (bottom) & $137 \mathrm{v}$ \\
\hline LXIVv (top) & $144 \mathrm{v}$ \\
\hline LXIVv (bottom) & $137 \mathrm{r}$ (pencil 136) \\
\hline LXVr (top) & $139 \mathrm{r}$ (pencil 138) \\
\hline LXVr (bottom) & $142 \mathrm{v}$ \\
\hline LXVv (top) & $139 \mathrm{v}$ \\
\hline LXVv (bottom) & $142 \mathrm{r}$ (pencil 141) \\
\hline LXVIr (top) & $2 \mathrm{r}$ \\
\hline LXVIr (bottom) & $7 \mathrm{v}$ \\
\hline LXVIv (top) & $2 \mathrm{v}$ \\
\hline LXVIv (bottom) & $7 \mathrm{r}$ \\
\hline LXVIIr (top) & $149 \mathrm{r}$ (pencil 148) \\
\hline LXVIIr (bottom) & $148 \mathrm{v}$ \\
\hline LXVIIv (top) & \\
\hline
\end{tabular}

\begin{tabular}{|l|l|}
\hline Undertext & Overtext \\
\hline LXVIIv (bottom) & $148 \mathrm{r}$ (pencil 147) \\
\hline $\begin{array}{l}\text { LXVIIIr Only stub of } \\
\text { original (top) half } \\
\text { left. }\end{array}$ & $173 \mathrm{v}$ \\
\hline LXVIIIr (bottom) & $172 \mathrm{r}$ (pencil 171) \\
\hline $\begin{array}{l}\text { LXVIIIv Only stub of } \\
\text { original (top) half } \\
\text { left. }\end{array}$ & $173 \mathrm{r}$ (pencil 172) \\
\hline LXVIIIv (bottom) & $172 \mathrm{v}$ \\
\hline LXIXr (top) & $96 \mathrm{v}$ \\
\hline LXIXr (bottom) & $89 \mathrm{r}$ (pencil 88) \\
\hline LXIXv (top) & $96 \mathrm{r}$ (pencil 95) \\
\hline LXIXv (bottom) & $89 \mathrm{v}$ \\
\hline LXXr (top) & $155 \mathrm{r}$ (pencil 154) \\
\hline LXXr (bottom) & $158 \mathrm{v}$ \\
\hline LXXv (top) & $155 \mathrm{v}$ \\
\hline LXXv (bottom) & $158 \mathrm{r}$ (pencil 157) \\
\hline LXXIr (top) & $62 \mathrm{r}$ (pencil 61) \\
\hline LXXIr (bottom) & $59 \mathrm{v}$ \\
\hline LXXIv (top) & $62 \mathrm{v}$ \\
\hline LXXIv (bottom) & $59 \mathrm{r}$ (pencil 58) \\
\hline LXXIIr (top) & $111 \mathrm{r}$ (pencil 110) \\
\hline LXXIIr (bottom) & $106 \mathrm{v}$ \\
\hline LXXIIv (top) & $111 \mathrm{v}$ \\
\hline LXXIIv (bottom) & $106 \mathrm{r}$ (pencil 105) \\
\hline LXXIIIr (top) & $168 \mathrm{r}$ (pencil 167) \\
\hline LXXIIIr (bottom) & $161 \mathrm{v}$ \\
\hline LXXIIIv (top) & $168 \mathrm{v}$ \\
\hline LXXIIIv (bottom) & $161 \mathrm{r}$ (pencil 160) \\
\hline LXXIVr (top) & $167 \mathrm{r}$ (pencil 166) \\
\hline LXXIVr (bottom) & $162 \mathrm{v}$ \\
\hline LXXIVv (top) & $167 \mathrm{v}$ \\
\hline LXXIVv (bottom) & 162 pencil 161) \\
\hline
\end{tabular}




\begin{tabular}{|l|l|}
\hline Undertext & Overtext \\
\hline LXXVr (top) & $112 \mathrm{r}$ (pencil 111) \\
\hline LXXVr (bottom) & $105 \mathrm{v}$ \\
\hline LXXVv (top) & $112 \mathrm{v}$ \\
\hline LXXVv (bottom) & $105 \mathrm{r}$ (pencil 104) \\
\hline LXXVIr (top) & $63 \mathrm{r}$ (pencil 62) \\
\hline LXXVIr (bottom) & $58 \mathrm{v}$ \\
\hline LXXVIv (top) & $63 \mathrm{v}$ \\
\hline LXXVIv (bottom) & $58 \mathrm{r}$ (pencil 57) \\
\hline LXXVIIr (top) & $84 \mathrm{r}$ (pencil 83) \\
\hline LXXVIIr (bottom) & $85 \mathrm{v}$ \\
\hline LXXVIIv (top) & $84 \mathrm{v}$ \\
\hline LXXVIIv (bottom) & $85 \mathrm{r}$ (pencil 84) \\
\hline LXXVIIIr (top) & $99 \mathrm{r}$ (pencil 98) \\
\hline LXXVIIIr (bottom) & $102 \mathrm{v}$ \\
\hline LXXVIIIv (top) & $99 \mathrm{v}$ \\
\hline LXXVIIIv (bottom) & $102 \mathrm{r}$ (pencil 101) \\
\hline LXXIXr (top) & $129 \mathrm{r}$ (pencil 128) \\
\hline LXXIXr (bottom) & $136 \mathrm{v}$ \\
\hline LXXIXv (top) & $129 \mathrm{v}$ \\
\hline LXXIXv (bottom) & $136 \mathrm{r}$ (pencil 135) \\
\hline LXXXr (top) & $12 \mathrm{r}$ \\
\hline LXXXr (bottom) & $13 \mathrm{v}$ \\
\hline LXXXv (top) & $12 \mathrm{v}$ \\
\hline LXXXv (bottom) & $13 \mathrm{r}$ \\
\hline LXXXIr (top) & $11 \mathrm{r}$ \\
\hline LXXXIr (bottom) & $14 \mathrm{v}$ \\
\hline LXXXIv (top) & $11 \mathrm{v}$ \\
\hline LXXXIv (bottom) & $14 \mathrm{r}$ \\
\hline LXXXIIr (top) & $130 \mathrm{r}$ (pencil 129) \\
\hline LXXXIIr (bottom) & $135 \mathrm{v}$ \\
\hline LXXXIIv (top) & $130 \mathrm{v}$ \\
\hline LXXIIv (bottom) & $135 \mathrm{r}$ (pencil 134) \\
\hline
\end{tabular}

\begin{tabular}{|c|c|}
\hline Undertext & Overtext \\
\hline LXXXIIIr (top) & $164 r($ pencil 163) \\
\hline LXXXIIIr (bottom) & $165 \mathrm{v}$ \\
\hline LXXXIIIv (top) & $164 \mathrm{v}$ \\
\hline LXXXIIIv (bottom) & 165r (pencil 164) \\
\hline LXXXIVr (top) & $\begin{array}{l}25 \mathrm{r} \text { (pencil } 24 \\
\text { corrected to } 25)\end{array}$ \\
\hline LXXXIVr (bottom) & $32 \mathrm{v}$ \\
\hline LXXXIVv (top) & $25 v$ \\
\hline LXXXIVv (bottom) & 32r (pencil 31) \\
\hline LXXXVr (top) & 26r (pencil 25) \\
\hline LXXXVr (bottom) & $31 \mathrm{v}$ \\
\hline LXXXVv (top) & $26 \mathrm{v}$ \\
\hline LXXXVv (bottom) & $31 \mathrm{r}$ (pencil 30) \\
\hline LXXXVIr (top) & 30r (pencil 29) \\
\hline LXXXVIr (bottom) & $27 \mathrm{v}$ \\
\hline LXXXVIv (top) & $30 v$ \\
\hline LXXXVIv (bottom) & $27 \mathrm{r}$ (pencil 26) \\
\hline LXXXVIIr (top) & 29r (pencil 28) \\
\hline LXXXVIIr (bottom) & $28 \mathrm{v}$ \\
\hline LXXXVIIv (top) & $29 \mathrm{v}$ \\
\hline LXXXVIIV (bottom) & $\begin{array}{l}28 \mathrm{r}(\text { pencil } 26 \\
\text { corrected to } 27)\end{array}$ \\
\hline LXXXVIIIr (top) & 171r (pencil 170) \\
\hline LXXXVIIIr (bottom) & $174 \mathrm{v}$ \\
\hline LXXXVIIIv (top) & $171 v$ \\
\hline LXXXVIIIv (bottom) & $174 \mathrm{r}$ (pencil 173) \\
\hline $\begin{array}{l}\text { LXXXIXr (bottom) } \\
\text { (top missing, sewn } \\
\text { onto VIIr) }\end{array}$ & $176 \mathrm{v}$ \\
\hline $\begin{array}{l}\text { LXXXIXv (bottom) } \\
\text { (top missing, sewn } \\
\text { onto VIIv) }\end{array}$ & 176r (pencil 175) \\
\hline
\end{tabular}

